\title{
AN EVALUATION MODEL TO REDUCE ANXIETY IN UNIVERSITY STUDENTS
}

\author{
$\underline{\text { Mg. Adriana D'Amelio }}{ }^{1}$, Lic. Eleonora Mamaní ${ }^{1}$, Lic. Johana Gisel Tari ${ }^{2}$ \\ ${ }^{1}$ National University of Cuyo, Mendoza, Argentina \\ ${ }^{2}$ University of Aconcagua Mendoza, Argentina \\ adriana.damelio@,fce.uncu.edu.ar
}

In the last few years, students of Quantitative Analysis of the Faculty of Political Sciences, have had many difficulties in issues related to the application of statistical concepts, which led to lack of motivation and withdrawal from the subject. Considering this problem, in 2018, a new methodology was implemented to evaluate students with the objective of understanding the importance of statistics in the work practice of their career and its applications. This new assessment achieves an improvement in academic performance by having students research and analyze a database, work in groups, and prepare an oral presentation. This new appraisal replaces the written test, which used to generate high levels of anxiety, and allows students to acquire other useful knowledge, such as managing databases, working in groups, using software, etc. Students' status is analyzed through the instrument used to measure the anxiety level GTAIA.

\section{INTRODUCTION}

Examination anxiety (EA) is defined as a predisposition to experience high concern and physiological activation in evaluation situations (Mandler \& Sarason, 1952). Given the negative characteristics of EA, teachers and researchers have been interested in proposing a new evaluation methodology that reduces anxiety before the test.

Academics are seen as a set of stressful situations in which people experience a lack of control over the situation and thus generate responses of academic stress and failure, which can affect both teachers and students. For such individuals, the academic environment raises stress-generating problems that they must confront with the resources they have (García-Ros, Pérez-González, Pérez-Blasco \& Natividad, 2012).

Therefore, it is not the examination situation that generates stress, but rather it is linked to the "oppression" that causes students the relationship between the amount of work/time, that is, when they perceive the amount of tasks to be done and the little time to carry them out (Polo, López, \& Muñoz, 1996). In the same way, it could be affirmed that there are stressors present in a university career, so that the examination situation constitutes one of the main sources of stress and tension for university students (Casari, Anglada, \& Daher, 2014).

\section{METHODOLOGY}

Students who are attending the Quantitative Analysis course, cohort 2019, of the Faculty of the Political Science and Public Administration at the National University of Cuyo, participated in this study.

The students are in the second year of the career of Political Science and Public Administration. Therefore, the analysis carried out is on the population of students who are studying this subject in the first semester of the current year (2019).

The methodology consisted of following the guidelines of the Descriptive and Inferential Statistics work uploaded to the virtual platform of the University 15 days before. They had to do it in groups of 3 students or less. There were 57 students in total.

The students presented the work in Power Point, the graphs and data analysis they did in Excel and also presented a written report. The exposition lasted between 20 and 30 minutes each team asking questions to each member. They had filled out the survey previously.

The instrument used to measure the level of test anxiety was the Argentine adaptation of the German test anxiety inventory (GTAI-A, Heredia, Piemontesi, Furlan, \& Volker, 2008), a 29-item selfreport measure $(\alpha$ for the full scale $=.92$ ) with five Likert-type response options ranging from $1="$ never" to 
5="always". This scale contemplates 4 dimensions: Concern (refers to thoughts about the negative consequences of poor performance), Interference (refers to cognitions that interfere with the assessment situation regardless of their specific content), Emotionality (describes perceptions of physiological activation experienced by the student), and Lack of Confidence (refers to the lack of confidence in one's own performance and self-control during the examination situation).

Following Furlan's work (2013), the distribution is as follows:

Scales of GTAI - A:

\begin{tabular}{|c|c|c|c|}
\hline Concern & Interference & $\begin{array}{c}\text { Lack of confidence } \\
\text { (inverse) }\end{array}$ & Emotionality \\
\hline $2,5,8,9,13,16,20,22,26$ & $4,10,14,18,23,29$ & $1,7,12,19,25,28$ & $3,6,11,15,17,21,24,27$ \\
\hline
\end{tabular}

It is important to clarify that the dimension of "lack of confidence" presents an inverted coding due to the positive nature of sentences such as "I have confidence in my capability".

In addition, the total score of the indicator of anxiety before the examination arises from the sum of the 4 scales.

To make the scale we used the percentiles $0.2,0.4,0.6,0.8$ of the scores obtained in the sum of the 4 dimensions and the total in order to compare the percentages by dimension and if these varied with respect to the total.

\section{RESULTS}

Based on the data analyzed from the measurement of the students' anxiety regarding. This new evaluation model, it was observed that $55 \%$ of the students have a level of anxiety regarding the average to low examination situation in the total score.

Table 1. Levels of anxiety in front of the examination in the course Quantitative Analysis UNCuyo, Mendoza

\begin{tabular}{lcclcr}
\hline & P & I & FC & E & Total \\
\hline Low & $23 \%$ & $23 \%$ & $25 \%$ & $26 \%$ & $18 \%$ \\
Medium Low & $23 \%$ & $19 \%$ & $18 \%$ & $14 \%$ & $19 \%$ \\
Medium & $19 \%$ & $19 \%$ & $25 \%$ & $25 \%$ & $18 \%$ \\
Medium high & $25 \%$ & $18 \%$ & $16 \%$ & $16 \%$ & $16 \%$ \\
High & $11 \%$ & $21 \%$ & $18 \%$ & $19 \%$ & $30 \%$ \\
\hline
\end{tabular}

In the four dimensions of anxiety evaluated $(\mathrm{P}=$ Concern, $\mathrm{I}=$ Interference $\mathrm{E}=$ Emotionality and $\mathrm{FC}=$ Lack of Confidence) the students present a $40 \%$ or more low and low average anxiety level. It should be noted that $70 \%$ of students in the Lack of Confidence dimension present medium to low anxiety. 


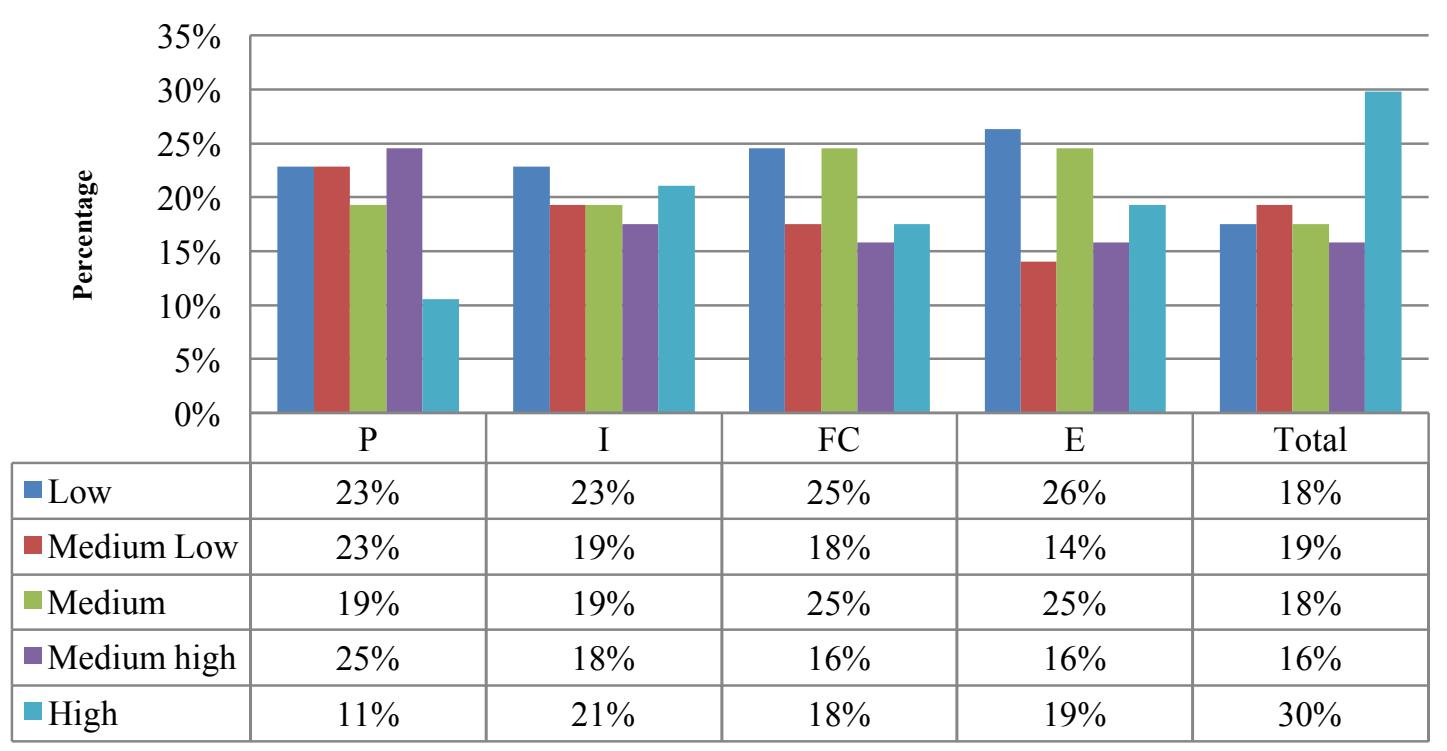

Fte : Own elaboration. Uncuyo. Mendoza. May 2019

Figure 1. Levels of anxiety in front of the exam in the course Quantitative Analysis UNCuyo, Argentina

\section{CONCLUSIONS}

Of the total number of students evaluated in 25 groups, 20 groups were approved. Although the total score shows a high anxiety of $30 \%$ of the students, this may be due to the fact that the subject conditions the future of their Career, since it is the correlative of other subjects, and generates distrust regarding whether or not they will be able to receive it.

While in the levels of anxiety the one who has the greatest incidence is the emotionality. This may be due to the fact that students, feeling uncomfortable with the sensation generated by taking the exam, decide either to give up the possibility of taking the exam, which causes their level of anxiety to decrease. This happened with several students, who did not want to take the subject because they felt sick, and then they were given the encouragement to face it and their performance levels were very good.

We can consider that a greater predisposition of the students to the new examination methodology was observed, which was manifested in their interest, by resorting to all the consultation classes, in which a large number of students attended, to consult on how to search databases, the pages in which databases can be obtained, and so on.

Besides, there was a greater commitment of the students, working in groups, since it was possible to see the equitable distribution of tasks among them. (Some were in charge of collecting data, others of organizing them, and others of representing them graphically, although always with total complementarity, since if someone in the group could not solve their task, they solved it all together).

Among the testimonies that can be cited from the students, it can be mentioned that although the great majority stated that this work demanded a lot of time, the learning they achieved by doing it was unmatched, many said that it was the first time they used the tool "Excel" and that for the first time they were able to interpret data and draw conclusions based on them. Some of those students used this analysis to present new projects in their work.

However, some consequences of these findings are relevant to the design of intervention programs. First, when a student with high test anxiety faces the challenge of returning to a subject in which he or she was suspended or must optimize the limited time available to prepare for an imminent test, the tendency to ruminate and/or assign blame, is likely to increase cognitive interference during study activities, disperse his or her attention, lead him or her to use his or her time in an inappropriate manner, and decrease his or her motivation to learn; creating conditions for his anxiety to increase and give up his 
attempt to give up, or end up doing so under a lot of pressure and in disadvantageous conditions. (Piemontesi et al., 2012)

Therefore, the inclusion of cognitive restructuring techniques that help to realistically assess the factors that led to eventual failure and to perceive the necessary corrective actions can contribute to replacing guilt assignments with a more proactive sense of responsibility, which also increases the sense of control over the learning process.

\section{IMPLICATIONS}

This new methodology improve the academic performance, the concurrence of students until the end of the course and the integration of the specific contents of the subject. Based on the results, this methodology will continue to be applied with the pertinent improvements.

\section{REFERENCES}

Casari L. M., Anglada, J., \& Daher, C. (2014). Coping strategies and exam anxiety in college students. Revista de Psicología, 32(2), 243-269

Furlan, L. (2013). Inventario Alemán de Ansiedad frente a los Exámenes: Valores normativos para su adaptación Argentina (GTAI-AR). Unpublished manuscript.

García-Ros, R., Pérez-González, F., Pérez-Blasco, J., \& Natividad, L. A. (2012). Evaluación del estrés académico en estudiantes de nueva incorporación a la universidad. Revista latino americana de psicología, 44(2), 143-154.

Heredia, D., Piemontesi, S., Furlan, L., \& Volker, H. (2008). Adaptación del Inventario Alemán de Ansiedad frente a los Exámenes: GTAI-A. Revista Evaluar, 8(1), 46-60.

Mandler, G., \& Sarason, S. B. (1952). A study of anxiety and learning. The Journal of Abnormal and Social Psychology, 47(2), 166-173.

Piemontesi, S. E., Heredia, D. E., Furlan, L. A., Sánchez Rosas, J., \& Martínez, M. (2012). Test anxiety and coping styles with academic stress in university students. Annals of Psychology, 28(1), 89-96.

Polo, A., López, J. M. H., \& Muñoz, C. P. (1996). Evaluación del estrés académico en estudiantes universitarios. Ansiedad y estrés, 2(2), 159-172. 